\title{
TP53 and RET may serve as biomarkers of prognostic evaluation and targeted therapy in hepatocellular carcinoma
}

\author{
SONG YE ${ }^{1 *}$, XIN-YI ZHAO ${ }^{*}$, XIAO-GE HU ${ }^{2}$, TANG LI $^{2}$, QIU-RAN XU² ${ }^{2}$ HUAN-MING YANG ${ }^{5}$, \\ DONG-SHENG HUANG ${ }^{2,3}$ and LIU YANG ${ }^{2,3}$
}

\begin{abstract}
${ }^{1}$ Division of Hepatobiliary and Pancreatic Surgery, Department of Surgery, The First Affiliated Hospital, School of Medicine, Zhejiang University, Hangzhou, Zhejiang 310003; ${ }^{2}$ Key Laboratory of Tumor Molecular Diagnosis and Individualized Medicine of Zhejiang Province, Key Laboratory of Gastroenterology of Zhejiang Province, Zhejiang Provincial People's Hospital, Hangzhou, Zhejiang 310014; ${ }^{3}$ Key Laboratory of Tumor Molecular Diagnosis and Individualized Medicine of Zhejiang Province, Key Laboratory of Gastroenterology of Zhejiang Province, People's Hospital of Hangzhou Medical College, Hangzhou, Zhejiang 310014; ${ }^{4}$ State Key Laboratory for Diagnosis and Treatment of Infectious Diseases, The First Affiliated Hospital, Collaborative Innovation Center for Diagnosis and Treatment of Infectious Diseases, Zhejiang University School of Medicine, Hangzhou, Zhejiang 310003;

${ }^{5}$ Beijing Genomics Institute (BGI)-Shenzhen, Shenzhen, Guangdong 518103, P.R. China
\end{abstract}

Received November 10, 2016; Accepted February 10, 2017

DOI: $10.3892 /$ or.2017.5494

\begin{abstract}
Hepatocellular carcinoma (HCC) is the most common malignancy of the liver. Genomic analysis is conducted to identify genetic alterations in driver genes which are all druggable targets for cancer therapy. In the present study, we performed an exome sequencing of 45 driver genes in 100 paired samples from HCC patients including tumors and matched adjacent normal tissues using Illumina HiSeq 2000 platform. Non-synonymous mutations were ascertained using the iPLEX MassARRAY system and Sanger sequencing. Clinicopathological relevance with genetic variations was assessed using SPSS software. The prognostic analyses of patients with gene mutation status were summarized using Kaplan-Meier curves. Sixty-one non-synonymous somatic mutations were identified in $43 \%$ of the HCC patients. The most frequent mutations were: TP53 (20\%), RET (6\%), PLCEI (5\%), PTEN (4\%) and VEGFR2 (3\%). Patients with mutations in TP53 had a lower overall survival (OS) $(\mathrm{P}=0.002)$ than those without mutations. Recurrent mutations in the Ret proto-oncogene (RET) were associated with poor outcomes for
\end{abstract}

Correspondence to: Professor Liu Yang or Professor Dong-Sheng Huang, Key Laboratory of Tumor Molecular Diagnosis and Individualized Medicine of Zhejiang Province, Key Laboratory of Gastroenterology of Zhejiang Province, Zhejiang Provincial People's Hospital, Shang Tang Road 158, Hangzhou, Zhejiang 310014, P.R. China

E-mail: yangliuqq2003@163.com

E-mail: dshuang@zju.edu.cn

${ }^{*}$ Contributed equally

Key words: hepatocellular carcinoma, TP53, RET, somatic mutation, targeted deep sequencing, molecular-targeted therapy both disease-free survival (DFS) $(\mathrm{P}=0.028)$ and $\mathrm{OS}(\mathrm{P}=0.001)$ in HCC patients. The mutational status of sorafenib-targeted genes were associated with decreased DFS $(\mathrm{P}=0.039)$, and decreased OS $(\mathrm{P}=0.15)$ without statistical significance. Mutual exclusion of TP53 and RET mutations were observed in the present study. In conclusion, patients with TP53 mutations, $R E T$ mutations and sorafenib-targeted gene mutations were demonstrated to be associated with poor HCC prognosis, which suggests that both TP53 and RET may serve as biomarkers of prognostic evaluation and targeted therapy in HCC.

\section{Introduction}

Hepatocellular carcinoma (HCC) is the most common malignancy of the liver. It is the fifth most common cancer in men and the seventh amongst women worldwide $(1,2)$. There is a high incidence of HCC in China due to hepatitis B virus (HBV) infection (3). More than $80 \%$ of HCC tumors are inoperable with poor prognosis, and only $10-20 \%$ of HCC patients undergo curative treatments $(4,5)$. Results from clinical trials show a lack of survival benefits following HCC treatment with chemotherapeutic agents and conventional drugs. Thus, effective and well-tolerated treatment strategies for advanced HCC are urgently needed (6).

Sorafenib, an oral multikinase inhibitor of $B R A F, R A F 1$, FLT3, KIT, VEGFR and PDGFR, has been approved for the treatment of advanced HCC. The Sorafenib HCC Assessment Randomized Protocol trial and the Asia-Pacific trial demonstrated that sorafenib improves the survival of patients with advanced HCC $(7,8)$. Since this major development in HCC treatment, a focus has been shifted to identify novel agents that target driver genes and key molecular pathways in hepatocarcinogenesis (9). Their findings include components of the RAS/RAF/mitogen-extracellular activated protein kinase (MEK)/extracellular signal-regulated kinase (ERK), 
and phosphatidylinositol 3-kinase (PI3K)/AKT/mammalian target of rapamycin (mTOR) pathways that regulate cell proliferation, apoptosis, and protein synthesis; receptor tyrosine kinases (RTKs), including the epidermal growth factor receptor $(E G F R), K I T, F L T 3$ and RET, which transmit growth factor signals to downstream intracellular pathways; and proangiogenic factors that bind VEGFR and PDGFR which induce angiogenic signaling via the RAS/RAF/MEK/ERK, PI3K/AKT/mTOR and Wnt signal transduction pathways $(6,10-12)$. A thorough understanding of the mutations of genes associated with molecular-targeted therapy is needed to screen compounds and antibodies effective in HCC treatment.

Targeted sequencing allows accurate analysis of multiple cancer genes (13-15). However, mutational profiling of driver genes in Chinese patients with HCC has not been reported, to date. In the present study, we detected multiple mutations in 45 genes in 100 patients with HCC using next-generation targeted sequencing. These genes were categorized according to the following biological processes or signaling pathways: RTKs, angiogenesis, RAS/RAF/MEK/ERK and $\mathrm{PI} 3 \mathrm{~K} / \mathrm{AKT} / \mathrm{mTOR}$. In particular, we identified numerous novel somatic mutations in the driver genes and further found that patients with TP53, RET and sorafenib-targeted gene mutations, were associated with poor HCC prognosis.

\section{Materials and methods}

Patients. We analyzed 100 patients who underwent HCC resection between November 2009 and December 2011. Patients were subjected to pathological assessment in order to establish histological diagnosis and tumor cellularity. Only patients with a pathological diagnosis of HCC and tumor nuclei $\geq 80 \%$ of the total cellular nuclei were included. The present study was approved by the Ethics Committee of The First Affiliated Hospital, School of Medicine, Zhejiang University (Hangzhou, China). Signed informed consent forms were obtained from patients before their participation in the present study.

Exon capture array and deep sequencing. A customized NimbleGen HD 2.1 Array was constructed using the SeqCap v2 software. Target sequence capturing was performed using the SeqCap EZ Reagent kit. The captured DNA was randomly fragmented into an average size of 200-300 bp, and both ends of the fragments were ligated with adaptors that bind to different index primers. The enriched DNA fragments were eluted from the array and amplified by ligation-mediated PCR. On average, we sequenced the target exon regions of each sample to a mean depth of $75 x$ using the Illumina HiSeq 2000 platform.

Genome mapping and mutation detection. Mapping and Assembly with Quality software (http://maq.sourceforge.net) was used to align the sequence reads to the referenced human genome (hg19). The parameters used for the alignment were as follows: i) maximum distance between sequences, 300 ; ii) maximum allowed sum of qualities for 2-paired reads, 70; and iii) number of mismatches in the first $24 \mathrm{bp}$ of mismatches. Single nucleotide variations (SNVs) of high quality were obtained using the following filtering parameters: i) SNVs with depth $\geq 5$; ii) consensus quality $\geq 30$; iii) 3-bp flanking quality $\geq 40$; iv) highest mapping quality $\geq 30$; and v) SNVs with variant depth $\geq 8$. We defined the variant depth as 8 based on the results of a previous study (13). The high quality SNVs were filtered using the dbSNP (v.132) and $1 \mathrm{~K}$ Genome databases to define the mutations (16).

MassARRAY and Sanger sequencing validation. Non-synonymous mutations were validated using the iPLEX MassARRAY system (Sequenom Inc., San Diego, CA, USA) and Sanger sequencing in HCC tumors and paired peritumoural liver tissues to discriminate somatic and germline mutations. Both the PCR and MassEXTEND ${ }^{\circledR}$ primers for each mutation were in silico designed using the MassARRAY Assay design 4.0 software. Multiplex PCR was performed using the GeneAmp PCR System 9700 Dual 384-Well Sample Block Module (Applied Biosystems, Foster City, CA, USA), followed by dephosphorylation, single-base extension, and desalting. The MassARRAY Nanodispenser RS1000 was used to spot reactions with the 384 SpectroCHIP, which was loaded into a MALDI-TOF mass spectrometer. Genotype calls by MassARRAY Type 4.0 were confirmed by examining the spectra for each assay and sample. Mutations not detected by the iPLEX MassARRAY were reconfirmed by Sanger sequencing.

Immunohistochemistry and tissue microarray. In patients with multinodular tumors, samples were obtained from the largest tumor. Rabbit anti-human monoclonal Ret antibody (EPR 2871, 1:500; Abcam, Cambridge, MA, USA) was used to detect the protein expression of RET. The intensity of RET was calculated based on mean area of positive staining. Tissues were incubated with primary rabbit anti-human monoclonal Ret antibody (EPR2871, 1:500; Abcam), then, treated with biotinylated goat anti-rabbit secondary antibodies. Antibodies were visualized using diaminobenzidine hydrogen peroxidase as the chromogen, and slides were counterstained with $0.5 \%$ hematoxylin. In addition, we analyzed another 90 independent samples to elucidate RET protein expression using tissue microarray. Matched 90 pairs of primary HCC samples and peritumoral liver tissues were used to prepare tissue microarray (Shanghai Biochip Co., Ltd., Shanghai, China) as previously described (17). The intensity of RET was classified into high expression and low expression based on the mean area of positive staining. High expression was defined as $\geq 40 \%$ staining of a tumor section, and low expression as $<40 \%$.

Statistical analysis. Statistical analyses were performed using SPSS software (version 21.0; SPSS, Inc., Chicago, IL, USA). Age, gender, tumor stage, number, size and grade, HBV infection, and $\alpha$-fetoprotein (AFP) were the covariates of clinical characteristics included in the model. Chi-square and Fisher's exact tests were applied to compare the frequencies between genetic and clinical variables. The prognosis analyses of patients with gene mutation status were summarized using Kaplan-Meier curves. Univariate disease-free survival (DFS) and overall survival (OS) analyses were carried out using log-rank tests, and multivariate analyses were conducted using Cox's proportional hazards model. 


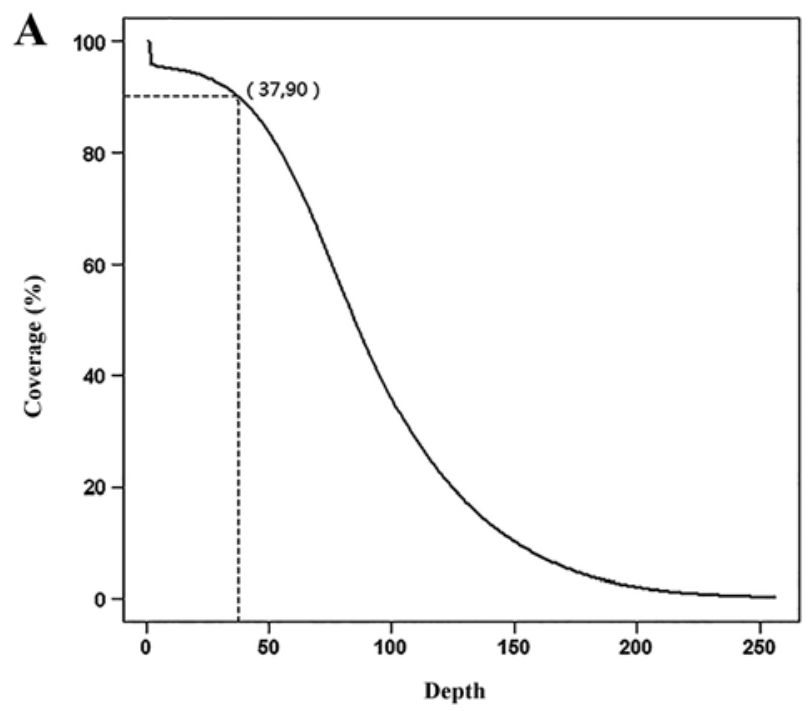

B

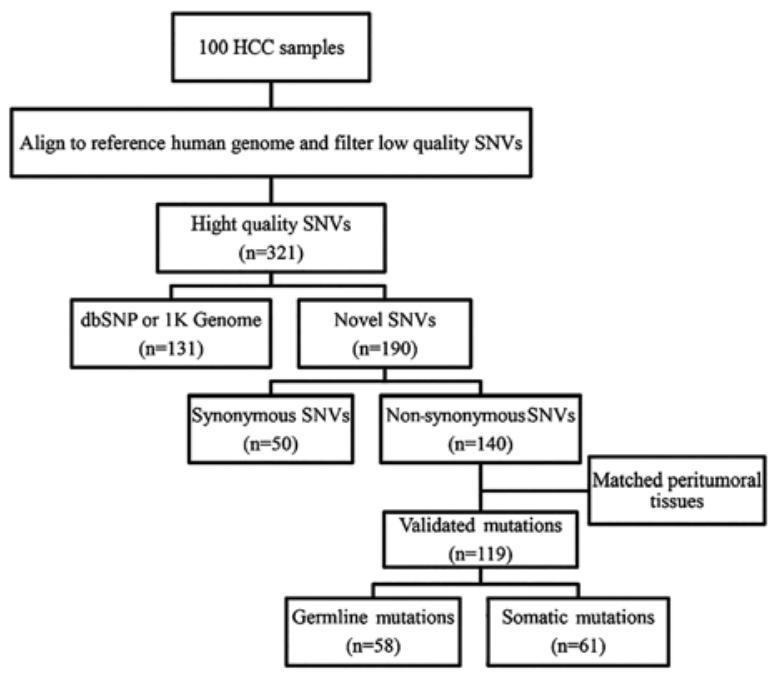

Figure 1. Overall cumulative coverage and flow-chart of the data analysis and mutation detection of genes in HCC patients. (A) A read coverage of $90 \%$ of the targeted exons was achieved as the sequencing depth was 37x. (B) In the cohort of $100 \mathrm{HCC}$ patients, 321 SNVs were detected by quality filters. After excluding single nucleotide polymorphisms in dbSNP and 1K Genome, 190 mutations remained of which 119 corresponded to non-synonymous mutations. After validation, 60 somatic mutations and 58 germline mutations were finally obtained. HCC, hepatocellular carcinoma; SNVs, single nucleotide variations.

Postoperative mortality was assessed, with deaths unrelated to tumor recurrence considered censored observations at the time of death. $\mathrm{P}<0.05$ was considered to indicate a statistically significant result.

\section{Results}

Genomic alteration landscape in HCC by whole-exome sequencing. Whole exons of 45 genes were sequenced in 100 patients with HCC using array-based sequence capture and Illumina HiSeq 2000 sequencing. Among them, 15 genes were associated with RTKs, 8 with angiogenesis, 13 with the RAS/RAF/MEK/ERK pathway, and 9 with the PI3K/AKT/mTOR pathway. For each sample, we generated an average of $33.75 \mathrm{Mb}$ bases, $97.9 \%$ of which were well-mapped to the human genome. The average sequencing depth was 75x (Table I). We achieved a read coverage of $90 \%$ of the targeted exons at the sequencing depth of 37x (Fig. 1A). These results indicate high quality targeted sequencing for mutation analysis.

SNV identification, validation and annotation. A computational pipeline was developed to discover novel SNVs (Fig. 1B). A total of 321 exonic SNVs were identified in the $100 \mathrm{HCC}$ patients. Approximately 40.8\% (131/321) of the SNVs were present after the data were filtered using the dbSNP and $1 \mathrm{~K}$ Genome databases. In most SNVs identified we observed genetic polymorphisms, which confirmed our sequencing data. The remaining 190 novel SNVs included 50 synonymous and 140 non-synonymous SNVs. In a random validation of 40 SNVs with MassARRAY and Sanger sequencing, the confirmation rate was $90 \%$. Furthermore, non-synonymous SNVs were validated in the original HCC and paired peritumoural liver tissues using MassARRAY and Sanger sequencing. We validated 119 mutations, of which 60 were somatic mutations and 58 were germline mutations. In the somatic mutations, 34 were novel mutations and 27 were
Table I. Summary of sequencing coverage of the $100 \mathrm{HCC}$ samples.

Mean of

Category

100 HCC samples

Total reads

$393,273.06$

Total bases

$35,394,575.4$

Average read length (bp)

90

Mappable reads

$385,309.01$

Mappable bases

$34,677,810.9$

Mappable base rates (\%)

97.90299419

Average sequencing depth

$75 \mathrm{x}$

Mean coverage over target gene

$75 \mathrm{x}$

HCC, hepatocellular carcinoma.

recorded in COSMIC, a public database of somatically acquired mutations in cancer (18).

The annotations of somatic mutations observed in the present study, are summarized in Table II. The list of functional domains (identified using the NCBI database) which harbor the mutations and molecular-targeted agents of the mutated genes were identified using the DrugBank database (Table II) (19). A few of the mutations were observed in the protein kinase domains, particularly the tyrosine kinase motifs of the target genes, which play a key role in the signaling pathways that contribute to carcinogenesis.

Mutation frequency of each gene distributed in 4 biological categories. A total of 60 somatic mutations occurred within 45 genes, with an average mutation frequency of $0.62 /$ affected individual. The number of somatic mutations ranged from 0 to 3 in the HCC patients. Fig. 2 shows the complete somatic mutation frequency of each gene in the 4 categories including 


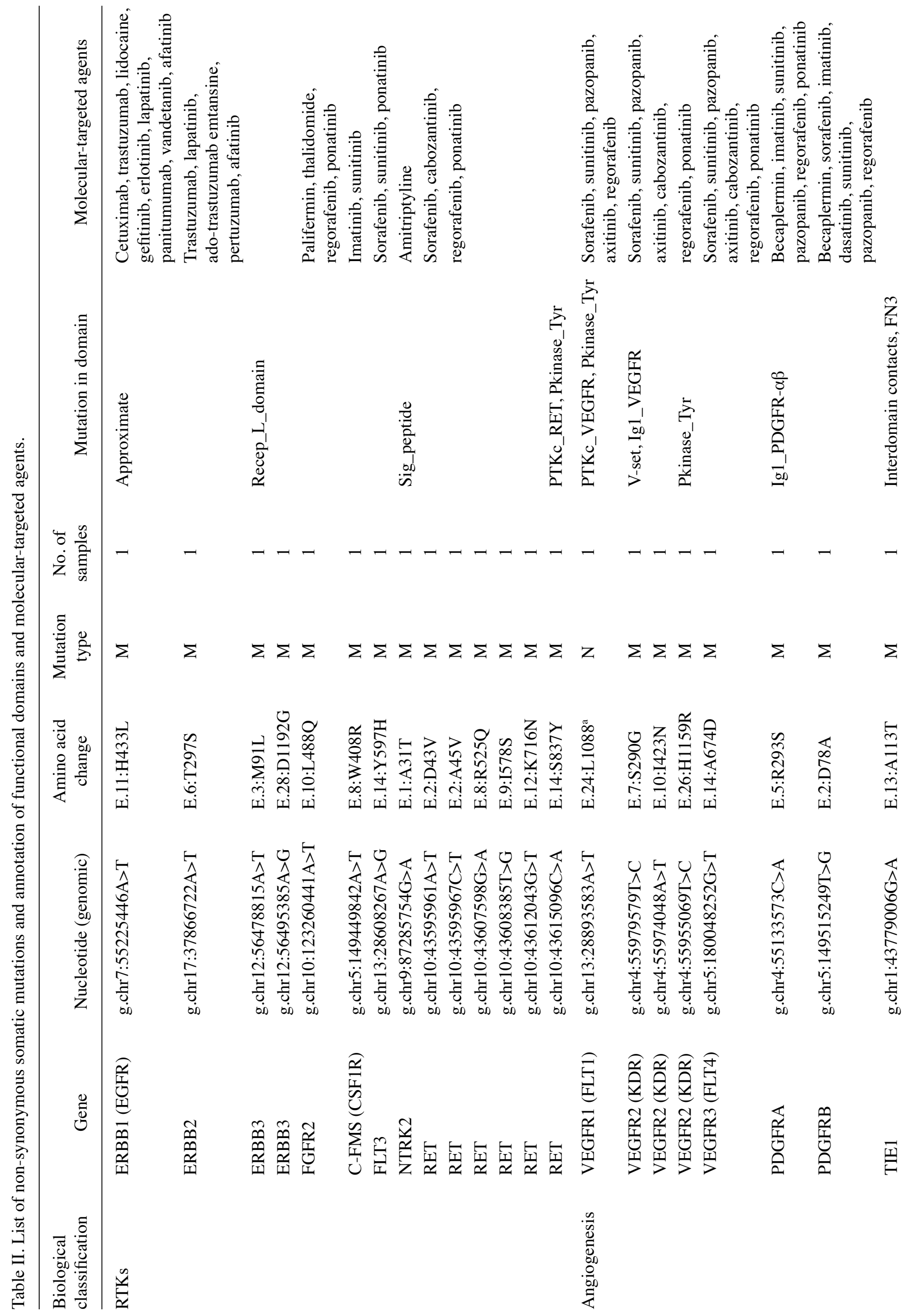




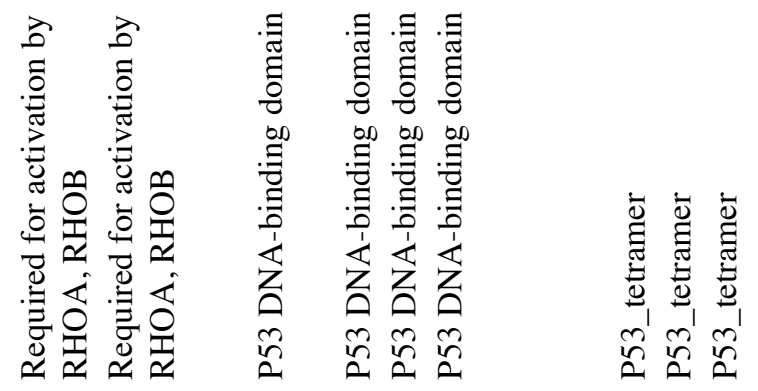

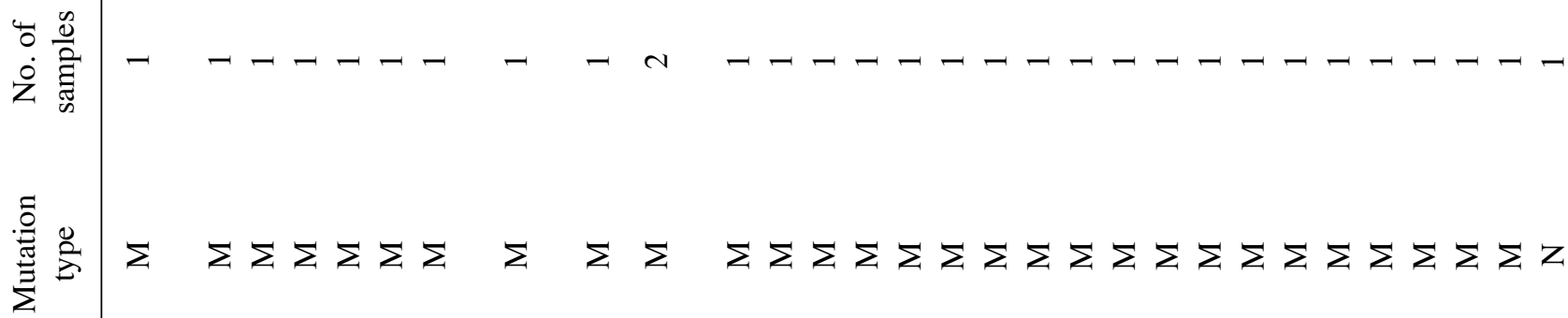

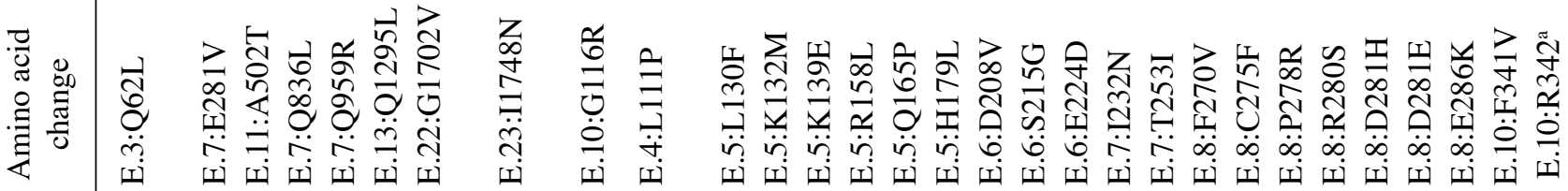

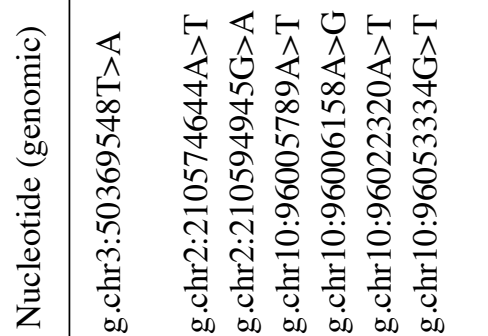

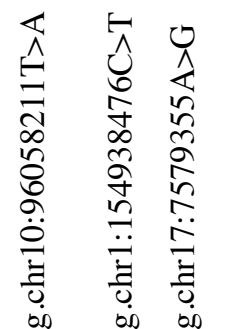

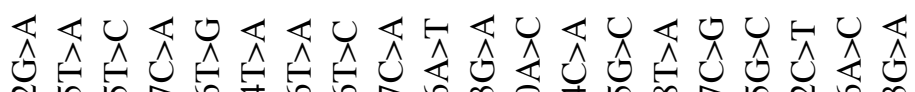

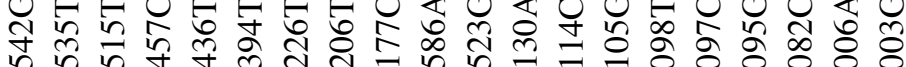

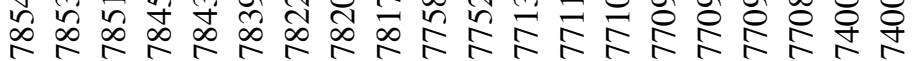

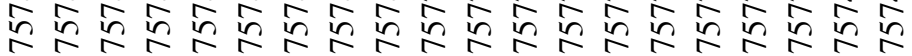

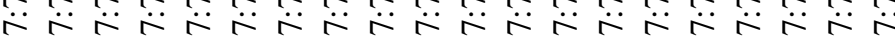

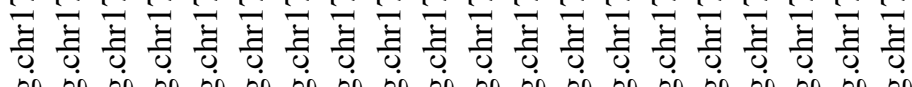

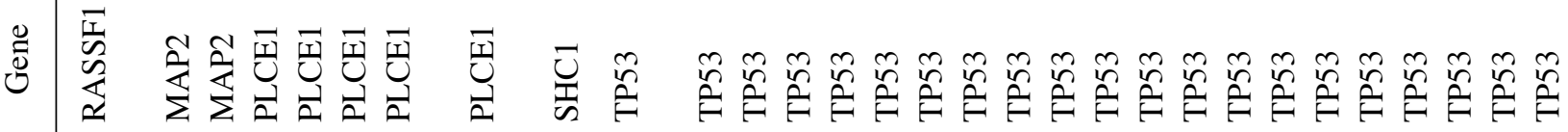




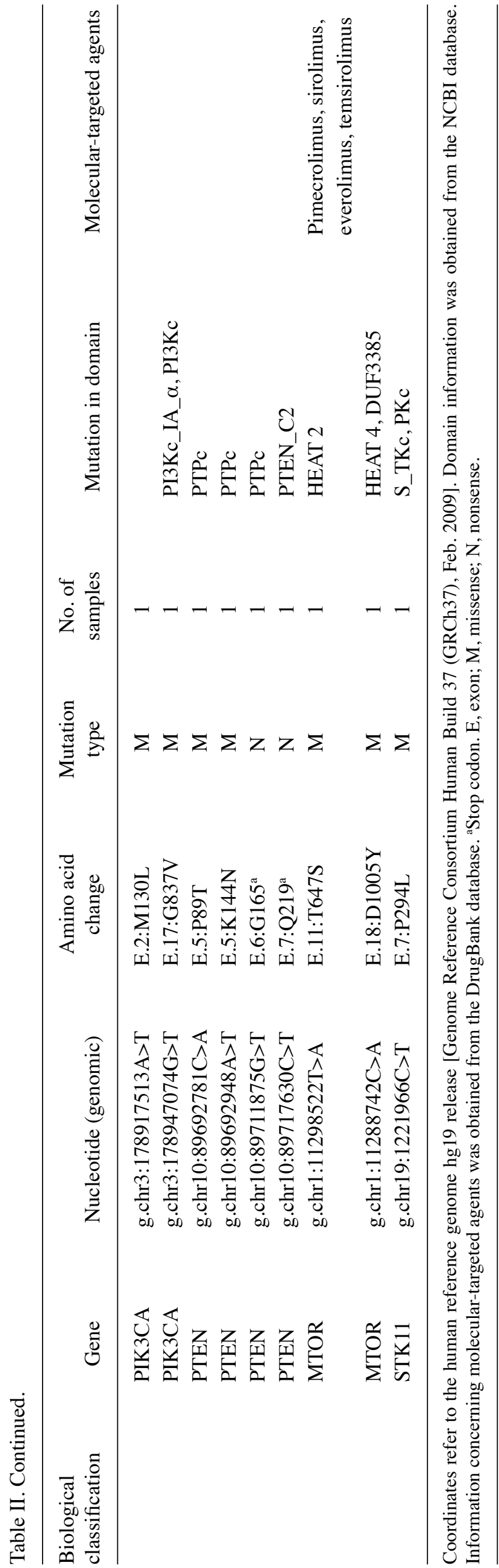

RTKs, angiogenesis, the RAS/RAF/MEK/ERK and the $\mathrm{PI} 3 \mathrm{~K} / \mathrm{AKT} / \mathrm{mTOR}$ pathways.

There were 14 somatic mutations in the RTK genes (Fig. 2), with 4 mutations identified in the EGFR family, including 1 in $E G F R, 1$ in ERBB2 and 2 in ERBB3. RET was found with a recurrent somatic mutation of $6 \%$ in $\mathrm{HCC}$, which was validated using MassARRAY and Sanger sequencing. The RET protein is composed of an extracellular ligand-binding domain, a hydrophobic transmembrane domain and a cytoplasmic part with a protein tyrosine kinase domain (TK domain) (20). Immunohistochemical staining of HCC with RET mutation further revealed a significant (Fig. 3A, the left 4 panels) or slight increase (Fig. 3A, the right 2 panels) in the expression of RET in tumor tissues compared with peritumoral tissues. We also performed a tissue microarray study of a cohort containing another 90 HCC patients. The protein expression tendency revealed that RET protein levels were higher in HCC tissues than in paired peritumoral liver tissues using Student's t-test $(\mathrm{P}=0.012$, Fig. 3B and $\mathrm{C})$. The group with the high expression of RET included $28.9 \%$ (26/90) of the patients. FGFR2, $C S F 1 R, F L T 3$ and NTRK2 had one mutation each, while there was no mutation for ERBB4, FGFR1, FGFR3, FGFR4, IGF1R, MET and KIT.

Eight somatic mutations were identified within genes associated with angiogenesis (Fig. 2). Of these mutations, 5 were identified in members of the VEGFR family, including one mutation in VEGFR1 (FLT1), 3 mutations in VEGFR2 (KDR), and 1 mutation in VEGFR3 (FLT4). PDGFRA, PDGFRB and TIEl had one mutation each, while no mutation was detected in PDGFRL and TEK (TIE2).

In the RAS/RAF/MEK/ERK pathway, 9 somatic mutations were detected (Fig. 2). Somatic mutations occurred mostly in PLCE1 (5\%). Another recurrent mutated gene was MAP2 (2\%), and $R A S S F 1$ and $S H C 1$ exhibited one somatic mutation each. No somatic mutation was observed in $E R K, R A F 1, A R A F$, $B R A F, N R A S, M E K 1, M E K 2, C X C R 4$ and KRAS.

Twenty-nine somatic mutations were detected within 9 genes associated with the PI3K/AKT/mTOR pathway, including recurrent mutations in TP53 (20\%), PTEN (4\%), mTOR (2\%), PIK3CA (2\%), and a single mutation in STK11 (Fig. 2). TP53 was the highest mutated gene. No somatic mutation was observed in $B A D, P D K 1, A K T 1$ and RPS6KB1.

Analysis of clinical characterization and prognosis. The clinicopathological characteristics of patients, including age, gender, tumor stage (American Joint Committee on Cancer; ver. 7), number, size, grade, serological HBV concentration and presence of the tumor marker AFP are summarized in Table III. The median follow-up of cases was 31.6 months (range, 1.8-48.1). A total of $39 \%$ of the patients died, with a median OS of 41.5 months and a 3-year OS of $64.0 \%$ as estimated by Kaplan-Meier analysis. During follow-up, 62 cases with recurrence were identified for a median DFS of 21.2 months. Correlation analysis of the clinical characteristics was based on our data. AFP-positive patients demonstrated a higher rate of $R E T$ mutations compared to those who were AFP-negative (11.1 vs. $0 \%$; $\mathrm{P}=0.039)$, without correlation to other genes.

The univariate analysis of DFS indicated that the significant predictors of DFS were the somatic mutation status of 


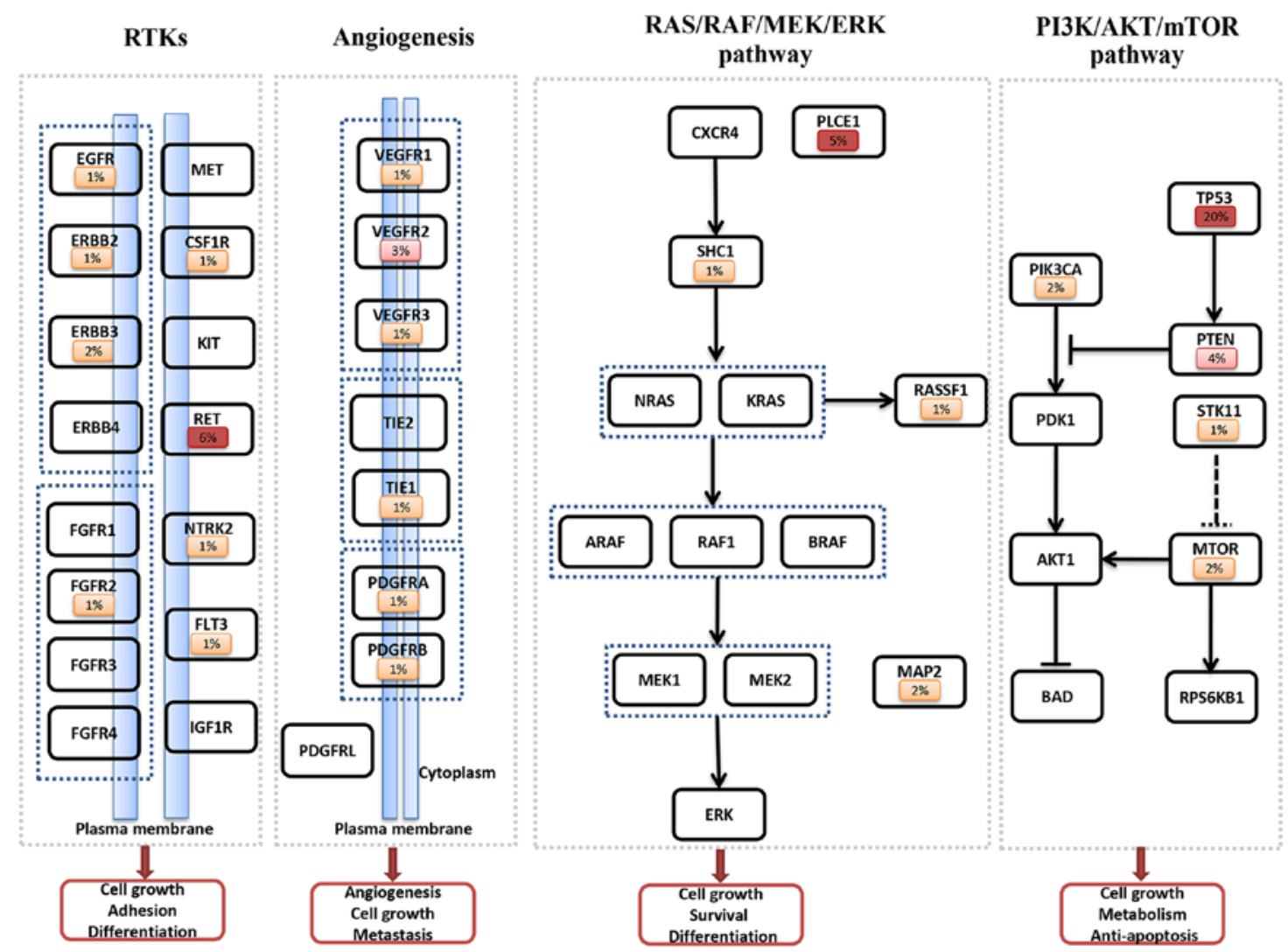

Figure 2. Mutation frequencies in 4 categories of molecular-targeted therapy-related genes. A total of 45 genes were classified into 4 categories, including RTKs, angiogenesis, the RAS/RAF/MEK/ERK and the PI3K/AKT/mTOR pathways. Genes are indicated in pink to red. The darkness of the color is positively correlated with the percentage of tumors with genetic alterations. The mutation frequency of each gene in 100 tumors is indicated. RTKs, receptor tyrosine kinases;MEK, mitogen-extracellular activated protein kinase; PI3K, phosphatidylinositol 3-kinase; mTOR, mammalian target of rapamycin; ERK, extracellular signal-regulated kinase.
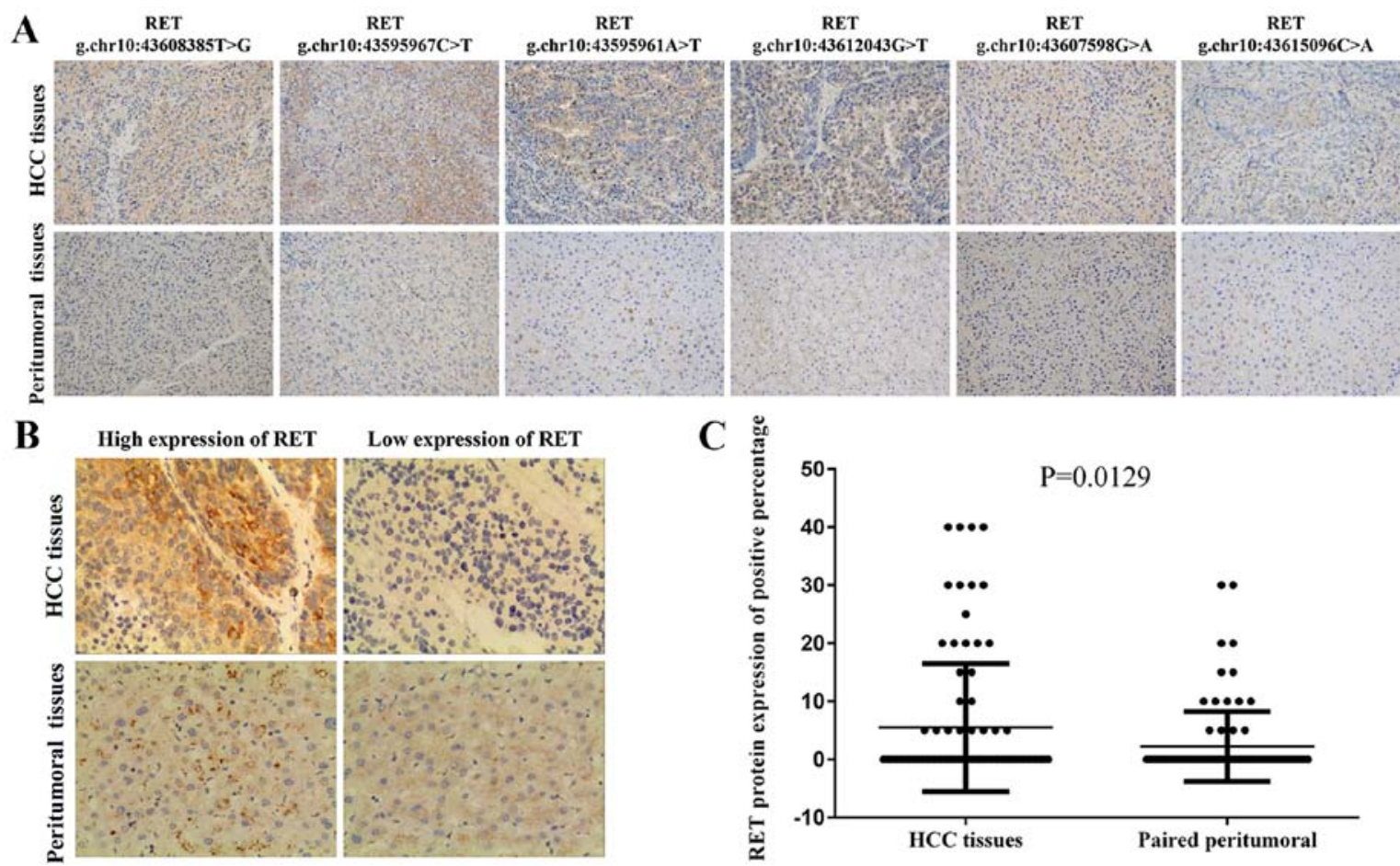

Figure 3. Expression of RET in HCC and peritumoral tissues with mutations in RET. (A) Immunohistochemical staining of HCC with RET mutation revealed significant (left 4 panels) or slight increase (right 2 panels) in the expression of RET in tumor tissues (upper panels) compared with peritumoral tissues (lower panels). (B) Representative expression of RET in HCC and paired peritumoral tissues using tissue microarray (magnification, x200). High expression is defined as $\geq 40 \%$ staining of the tumor section, and low expression as $<40 \%$. (C) Statistical results of RET expression in HCC and paired peritumoral tissues using Student's t-test $(\mathrm{P}=0.0129)$. HCC, hepatocellular carcinoma. 
Table III. Patient characteristics.

Factors

Total no. of patients $(\mathrm{N}=100)$

\begin{tabular}{lc} 
Age, years & \\
Median & 55 \\
Standard deviation & 11 \\
Gender & \\
Male & 84 \\
Female & 16 \\
HBV-DNA & \\
Positive & 64 \\
Negative & 36 \\
Stage & \\
I & 65 \\
II & 23 \\
IIIA & 7 \\
IIIB & 4 \\
IIIC & 1 \\
AFP (ng/ml) & \\
Positive $>20$ & 60 \\
Negative $\leq 20$ & 40 \\
Tumor grade (differentiation) & \\
Well & \\
Moderately & 50 \\
Poorly & \\
Tumor number & \\
Solitary & \\
Multifocal & \\
Tumor size (cm) & \\
Median & \\
Standard deviation & \\
\hline
\end{tabular}

The clinical staging of the tumors was according to the TNM classification system of the AJCC (edition 7). TNM, tumor-node-metastasis; AJCC, American Joint Committee on Cancer; HBV, hepatitis B virus; AFP, $\alpha$-fetoprotein.

RET $(\mathrm{P}=0.028)$, tumor size $(\mathrm{P}<0.001)$, tumor stage $(\mathrm{P}<0.001)$, and tumor marker AFP concentration $(\mathrm{P}=0.030)$ (Table IV and Fig. 4A). The somatic mutation status of TP53 was associated with decreased DFS without statistical significance (Table IV and Fig. 4C). Meanwhile, the univariate analysis of OS suggested that the somatic mutation status of RET $(\mathrm{P}=0.001)$ and TP53 $(\mathrm{P}=0.002)$, tumor size $(\mathrm{P}=0.002)$, tumor stage $(\mathrm{P}=0.009)$ and AFP concentration $(\mathrm{P}=0.007)$ were associated with the OS obtained from the follow-up (Table IV, Fig. 4B and D). Furthermore, the mutation status of sorafenib-target genes were associated with decreased DFS $(\mathrm{P}=0.039)$ and decreased OS $(\mathrm{P}=0.15)$ without statistical significance, which suggest poor prognosis in these patients (Fig. 4E and F).

The conditional multivariable analysis of DFS revealed that the somatic mutation status of RET (hazard ratio $(\mathrm{HR})=3.592$; 95\% confidence interval $(\mathrm{CI}), 1.331-9.693 ; \mathrm{P}=0.012)$, age of patients ( $\mathrm{HR}=1.029 ; 95 \% \mathrm{CI}, 1.003-1.055 ; \mathrm{P}=0.027)$, tumor size $(\mathrm{HR}=1.090 ; 95 \% \mathrm{CI}, 0.999-1.188 ; \mathrm{P}=0.053)$, tumor stage $(\mathrm{HR}=1.624 ; 95 \% \mathrm{CI}, 1.254-1.101 ; \mathrm{P}<0.001)$, and tumor marker AFP concentration $(\mathrm{HR}=1.000 ; 95 \% \mathrm{CI}, 1.000-1.000$; $\mathrm{P}=0.034$ ) (Table IV) were significant predictors of DFS. Conditional multivariable survival analysis demonstrated that the independent predictors of OS were the somatic mutation status of TP53 (HR=4.101; 95\% CI, 1.941-8.668; $\mathrm{P}<0.001)$, RET $(\mathrm{HR}=4.270 ; 95 \% \mathrm{CI}, 1.511-12.066 ; \mathrm{P}=0.006)$, and tumor size $(\mathrm{HR}=1.145 ; 95 \% \mathrm{CI}, 1.042-1.258 ; \mathrm{P}=0.005)$ (Table IV). In addition, mutual exclusion of TP53 and RET mutations was observed in the present study (Fig. 4G). These results suggest that both TP53 and RET are significant biomarkers in the prognosis of HCC.

\section{Discussion}

To date, sorafenib, an oral multi-kinase inhibitor of $B R A F$, $R A F 1, F L T 3, K I T, V E G F R$ and PDGFR, is the main clinical treatment used in advanced HCC. With the development of findings in new target driver genes and molecular-targeted therapies in HCC, RTKs, angiogenesis, RAS/RAF/MEK/ERK and $\mathrm{PI} 3 \mathrm{~K} / \mathrm{AKT} / \mathrm{mTOR}$ pathways have been reported to be involved in hepatocarcinogenesis (6,10-12). However, mutational profiling of these driver genes in Chinese patients with $\mathrm{HCC}$ has not been reported, to date. In the present study, targeted deep sequencing was used to conduct the simultaneous analysis of 45 driver genes in 100 patients with HCC, which were categorized according to the following biological processes or signaling pathways: RTKs, angiogenesis, RAS/RAF/MEK/ERK and PI3K/AKT/mTOR. To the best of our knowledge, this is the first comprehensive analysis of driver genes in Chinese patients with HCC.

In the present study, 61 non-synonymous somatic mutations were identified in $43 \%$ of the HCC patients. Among members of RTKs, we found somatic mutations in the EGFR family, of which FLT3, C-FMS and FGFR2 are the targets of multikinase inhibitors, including sorafenib, sunitinib and regorafenib, which exhibit effective results in HCC (21). Somatic mutations were also observed in angiogenesis-associated genes including VEGFR1, VEGFR2, VEGFR3, PDGFRA and PDGFRB, particularly non-synonymous mutations of L1088* (VEGFRI) and H1159R (VEGFR2) located in the catalytic domain of TK. Sorafenib targets both VEGFR2 and VEGFR3 with encouraging outcomes in advanced $\operatorname{HCC}(7,22)$. The dual inhibition of VEGF and PDGF signaling demonstrated marked anti-angiogenic effects in vivo (23). Linifanib (ABT-869) is a novel selective inhibitor of VEGF and PDGF RTK families in a phase III clinical trial for HCC treatment (24). Recurrent mutations in PLCE1 were also observed, since the RAS/RAF/MEK/ERK pathway participates in HCC growth and progression (6). Moreover, several studies have reported that the genetic variations in PLCEl are associated with esophageal squamous cell carcinoma and gastric adenocarcinoma $(25,26)$. Thus, PLCE1 may be further studied for HCC treatment. In addition, mutations of PIK3CA, PTEN and $m T O R$ in the $\mathrm{PI} 3 \mathrm{~K} / \mathrm{AKT} / \mathrm{mTOR}$ signaling pathway were observed. $P I K 3 C A$ mutations have been reported to sensitize cancer cells to mTOR inhibitor everolimus (27). In the present study, in our findings, somatic mutation of G837V was identified in the catalytic 
A

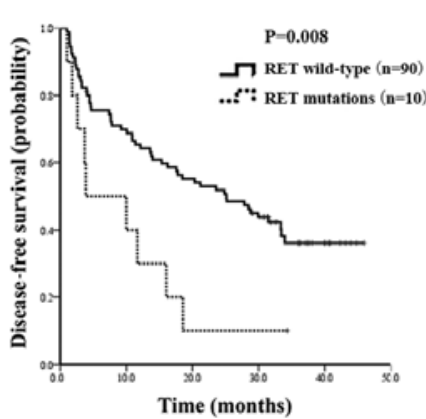

C

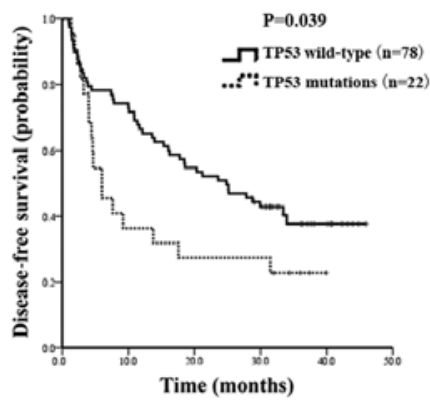

E

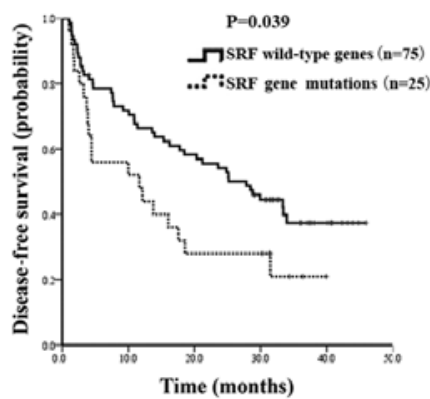

B

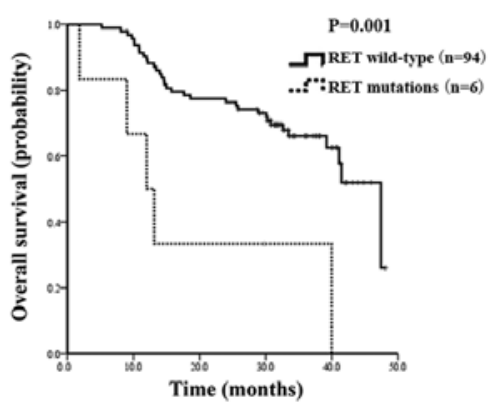

D

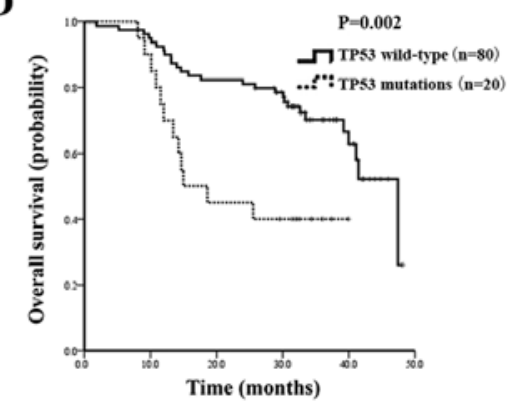

F

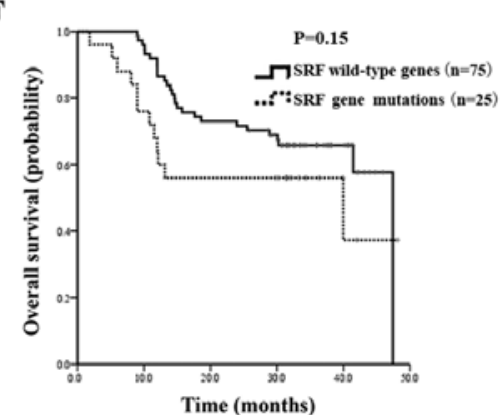

G

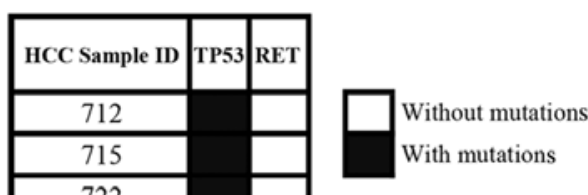

Figure 4. Kaplan-Meier survival estimates according to any mutations in TP53, RET and sorafenib-target genes, and mutation status of TP53 and RET in HCC patients. (A) Data are shown for the DFS of patients with and without mutations in RET (median DFS, 3.700 vs. 24.833 months, respectively, P=0.028). (B) Data are shown for the OS of patients with and without mutations in RET (median OS, 12.000 vs. 47.433 months, respectively, $\mathrm{P}=0.001$ ). (C) Data are shown for the DFS of patients with and without mutations in TP53 (median DFS, 24.172 vs. 24.833 months, respectively, $\mathrm{P}=0.133$ ). (D) Data are shown for the OS of patients with and without mutations in TP53 (median OS, 14.967 vs. 47.433 months, respectively, $\mathrm{P}=0.002$ ). (E) Data are shown for the DFS (median DFS, 11.667 vs. 27.833 months, respectively, $\mathrm{P}=0.039$ ) of patients with and without mutations in sorafenib-target genes. (F) Data are shown for the OS (median OS, 39.967 vs. 47.433 months, respectively, $\mathrm{P}=0.15$ ) of patients with and without mutations in sorafenib-target genes. (G) Mutations of TP53 and RET are mutually exclusive. Black indicates patients with mutations and white indicates patients without mutations. SRF, sorafenib; HCC, hepatocellular carcinoma; DFS, disease-free survival; OS, overall survival.

subunit of PIK3CA, and both G165* and Q219* mutations of $P T E N$ were truncating mutations which destroyed the function of PTEN. PTEN (G165*, Q219*) mutations may serve as molecular markers for mTOR inhibitor-targeted therapy.

As shown in Fig. 2, the most frequent mutations were: TP53 (20\%), RET (6\%), PLCE1 (5\%), PTEN (4\%) and VEGFR2 $(3 \%)$. Genome-wide sequencing analyses have revealed many mutant genes, such as TP53 and $\beta$-catenin $(C T N N B 1)$ in HCC $(28,29)$. The recurrent mutations of TP53 (20\%) identified in the present study were similar with earlier studies, which confirmed the reliability of our sequencing data. Further prognostic analysis which revealed that patients with mutations in TP53 had lower overall survival (OS) than those without mutations was consistent with earlier studies $(28,30)$. Dysregulation of RET activity is an important contributor to several human types of cancer including thyroid, lung, breast and pancreatic tumors (31-34), suggesting that RET is an important target for therapeutic intervention in many diseases (35). Genetic aberrations, including rearrangement, germline and somatic activation mutations, are responsible for a fraction of papillary thyroid carcinoma, medullary thyroid carcinoma, and a small subset of non-small cell lung cancer (7,32,36-38). In addition, the prognostic study further revealed that HCC patients with RET somatic mutations had poorer DFS and OS compared with wild-type patients in the present study. In particular, the mutual exclusion of TP53 and RET mutations were observed. All aforementioned results indicated that both TP53 and RET are significant biomarkers in the prognosis of HCC.

With the development of genome technology and lower costs for sequencing, the simultaneous analysis of genetic variation of the set of driver genes in tumor tissue after resection or biopsy is available. Presently, personalized medicine is emerging with genomic technology applied in clinical oncology. In addition, finding subpopulations of 


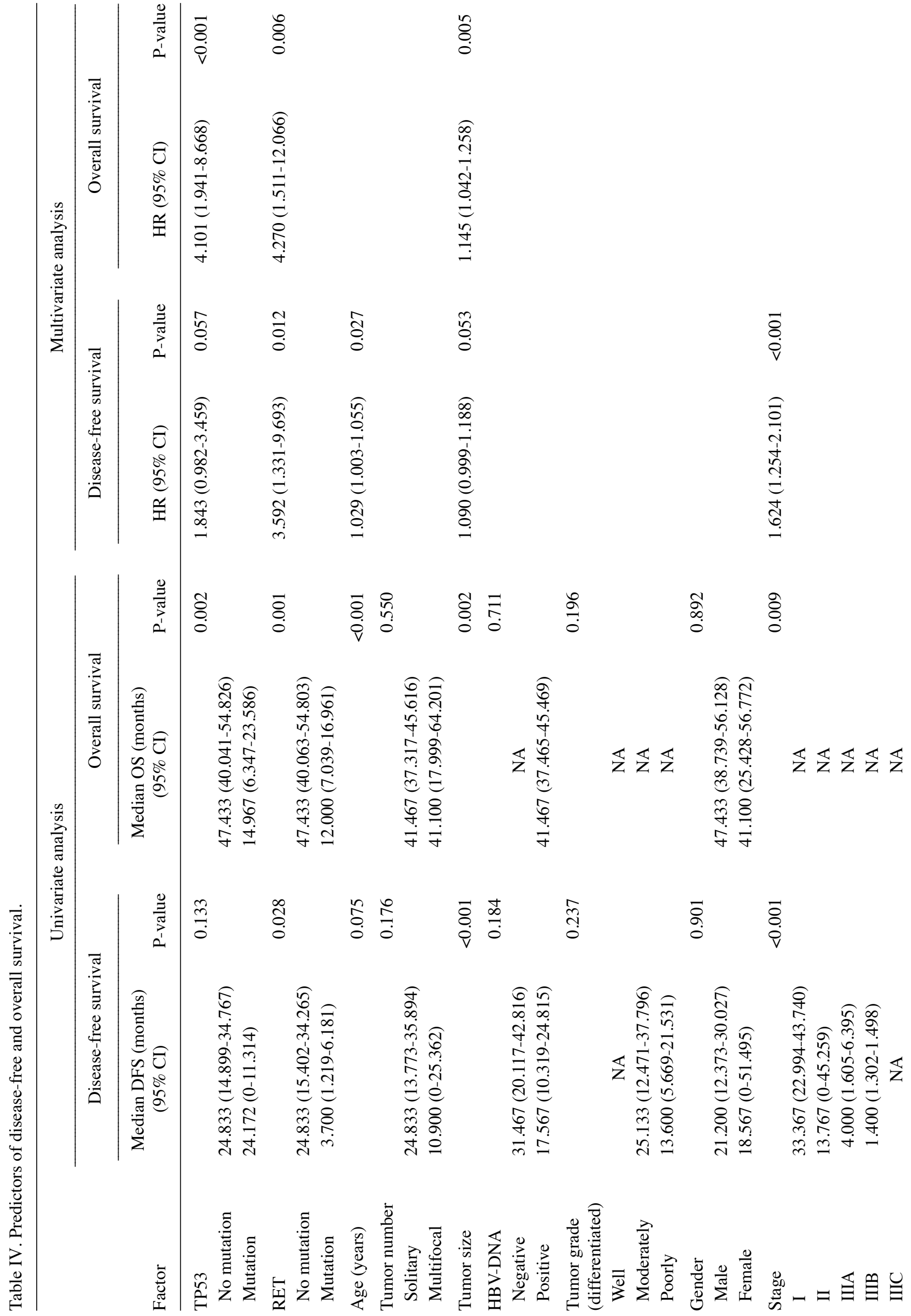


patients who may benefit from molecular-targeted therapy is important. Identification of specific genetic variations in individual patients may serve as a guide to develop effective drugs used in the treatment of HCC patients. In the present study, we conducted the mutation profiling of driver genes and clinical prognostic analysis, which indicated that TP53 and $R E T$ mutations may serve as biomarkers for targeted therapy in HCC. However, the present study has certain limitations. Firstly, we only analyzed a small group of patients, and a perspective analysis of frequent mutations may be carried out in a large-scale study of patients with HCC to confirm our results. Secondly, numerous genetic mutations were identified; however, not all mutations were functional, and may serve as passenger mutations. Thus, functional analysis of mutations is needed to further illustrate the carcinogenic mechanism in HCC. Although, further studies are needed to guide moleculartargeted therapy in HCC, in the present study we identified TP53 and RET mutations to be suitable markers for prognostic evaluation and targeted therapy in HCC.

\section{Acknowledgements}

We would like to thank Leiming Chen and Guoqiang Cao for their expert technical assistant in specimen collection. The present study was supported by the National High Technology Research and Development Program of China (863 Program, no. 2012AA02A205), the Science Technology Department of Zhejiang Province (nos. 2016C33116 and 2011C23088), the National Natural Science Fundation of China (no. J20121214), the CSCO Merck Serono Oncology Research Fund, SCORE (no. Y-MX2015-038), the Health Bureau of Zhejiang Province (no. 201484382), and the Key Research Project of Science Technology Department of Zhejiang Province (no. 2015C03030).

\section{References}

1. Mittal S and El-Serag HB: Epidemiology of hepatocellular carcinoma: Consider the population. J Clin Gastroenterol 47 (Suppl): S2-S6, 2013.

2. El-Serag HB: Epidemiology of viral hepatitis and hepatocellular carcinoma. Gastroenterology 142: 1264-1273, 2012.

3. Tanaka M, Katayama F, Kato H, Tanaka H, Wang J, Qiao YL and Inoue M: Hepatitis B and C virus infection and hepatocellular carcinoma in China: A review of epidemiology and control measures. J Epidemiol 21: 401-416, 2011.

4. Ferlay J, Shin HR, Bray F, Forman D, Mathers C and Parkin DM: Estimates of worldwide burden of cancer in 2008: GLOBOCAN 2008. Int J Cancer 127: 2893-2917, 2010.

5. Rampone B, Schiavone B, Martino A, Viviano C and Confuorto G: Current management strategy of hepatocellular carcinoma. World J Gastroenterol 15: 3210-3216, 2009.

6. Villanueva A and Llovet JM: Targeted therapies for hepatocellular carcinoma. Gastroenterology 140: 1410-1426, 2011.

7. Cheng AL, Kang YK, Chen Z, Tsao CJ, Qin S, Kim JS, Luo R, Feng J, Ye S, Yang TS, et al: Efficacy and safety of sorafenib in patients in the Asia-Pacific region with advanced hepatocellular carcinoma: A phase III randomised, double-blind, placebo-controlled trial. Lancet Oncol 10: 25-34, 2009.

8. Germano D, Tinessa V, Barletta E, Cannella L and Daniele B: Targeted therapy for advanced hepatocellular cancer in the elderly: Focus on sorafenib. Drugs Aging 30: 887-892, 2013.

9. Zhu AX: Molecularly targeted therapy for advanced hepatocellular carcinoma in 2012: Current status and future perspectives. Semin Oncol 39: 493-502, 2012.

10. Kudo M: Current status of molecularly targeted therapy for hepatocellular carcinoma: Clinical practice. Int J Clin Oncol 15: 242-255, 2010. 
11. Tanaka S and Arii S: Current status of molecularly targeted therapy for hepatocellular carcinoma: Basic science. Int J Clin Oncol 15: 235-241, 2010.

12. Finn RS: Emerging targeted strategies in advanced hepatocellular carcinoma. Semin Liver Dis 33 (Suppl 1): S11-S19, 2013

13. Zang ZJ, Ong CK, Cutcutache I, Yu W, Zhang SL, Huang D, Ler LD, Dykema K, Gan A, Tao J, et al: Genetic and structural variation in the gastric cancer kinome revealed through targeted deep sequencing. Cancer Res 71: 29-39, 2011.

14. Nikiforova MN, Wald AI, Roy S, Durso MB and Nikiforov YE: Targeted next-generation sequencing panel (ThyroSeq) for detection of mutations in thyroid cancer. J Clin Endocrinol Metab 98: E1852-E1860, 2013.

15. Cottrell CE, Al-Kateb H, Bredemeyer AJ, Duncavage EJ, Spencer DH, Abel HJ, Lockwood CM, Hagemann IS, O'Guin SM, Burcea LC, et al: Validation of a next-generation sequencing assay for clinical molecular oncology. J Mol Diagn 16: 89-105, 2014.

16. Abecasis GR, Auton A, Brooks LD, DePristo MA, Durbin RM, Handsaker RE, Kang HM, Marth GT and McVean GA; 1000 Genomes Project Consortium: An integrated map of genetic variation from 1,092 human genomes. Nature 491: 56-65, 2012.

17. Zhu XD, Zhang JB, Zhuang PY, Zhu HG, Zhang W, Xiong YQ, Wu WZ, Wang L, Tang ZY and Sun HC: High expression of macrophage colony-stimulating factor in peritumoral liver tissue is associated with poor survival after curative resection of hepatocellular carcinoma. J Clin Oncol 26: 2707-2716, 2008.

18. Forbes SA, Bindal N, Bamford S, Cole C, Kok CY, Beare D, Jia M, Shepherd R, Leung K, Menzies A, et al: COSMIC: Mining complete cancer genomes in the Catalogue of Somatic Mutations in Cancer. Nucleic Acids Res 39: D945-D950, 2011.

19. Knox C, Law V, Jewison T, Liu P, Ly S, Frolkis A, Pon A, Banco K, Mak C, Neveu V, et al: DrugBank 3.0: A comprehensive resource for 'omics' research on drugs. Nucleic Acids Res 39: D1035-D1041, 2011.

20. Fujimoto A, Totoki Y, Abe T, Boroevich KA, Hosoda F, Nguyen $\mathrm{HH}$, Aoki M, Hosono N, Kubo M, Miya F, et al: Whole-genome sequencing of liver cancers identifies etiological influences on mutation patterns and recurrent mutations in chromatin regulators. Nat Genet 44: 760-764, 2012.

21. Dekervel J, van Pelt J and Verslype C: Advanced unresectable hepatocellular carcinoma: New biologics as fresh ammunition or clues to disease understanding? Curr Opin Oncol 25: 409-416, 2013.

22. Llovet JM, Ricci S, Mazzaferro V, Hilgard P, Gane E, Blanc JF, de Oliveira AC, Santoro A, Raoul JL, Forner A, et al; SHARP Investigators Study Group: Sorafenib in advanced hepatocellular carcinoma. N Engl J Med 359: 378-390, 2008.

23. Kuhnert F, Tam BY, Sennino B, Gray JT, Yuan J, Jocson A Nayak NR, Mulligan RC, McDonald DM and Kuo CJ: Soluble receptor-mediated selective inhibition of VEGFR and PDGFRbeta signaling during physiologic and tumor angiogenesis. Proc Natl Acad Sci USA 105: 10185-10190, 2008.

24. Chiu YL, Carlson DM, Pradhan RS and Ricker JL: Exposure-response (safety) analysis to identify linifanib dose for a Phase III study in patients with hepatocellular carcinoma. Clin Ther 35: 1770-1777, 2013.

25. Bye H, Prescott NJ, Lewis CM, Matejcic M, Moodley L, Robertson B, Rensburg C, Parker MI and Mathew CG: Distinct genetic association at the PLCE1 locus with oesophageal squamous cell carcinoma in the South African population. Carcinogenesis 33: 2155-2161, 2012.
26. Palmer AJ, Lochhead P, Hold GL, Rabkin CS, Chow WH, Lissowska J, Vaughan TL, Berry S, Gammon M, Risch $\mathrm{H}$, et al: Genetic variation in C20orf54, PLCE1 and MUC1 and the risk of upper gastrointestinal cancers in Caucasian populations. Eur J Cancer Prev 21: 541-544, 2012.

27. Di Nicolantonio F, Arena S, Tabernero J, Grosso S, Molinari F, Macarulla T, Russo M, Cancelliere C,Zecchin D, Mazzucchelli L, et al: Deregulation of the PI3K and KRAS signaling pathways in human cancer cells determines their response to everolimus. J Clin Invest 120: 2858-2866, 2010.

28. Cleary SP, Jeck WR, Zhao X, Chen K, Selitsky SR, Savich GL, Tan TX, Wu MC, Getz G, Lawrence MS, et al: Identification of driver genes in hepatocellular carcinoma by exome sequencing. Hepatology 58: 1693-1702, 2013.

29. Kan Z, Zheng H, Liu X, Li S, Barber TD, Gong Z, Gao H, Hao K, Willard MD, Xu J, et al: Whole-genome sequencing identifies recurrent mutations in hepatocellular carcinoma. Genome Res 23: 1422-1433, 2013.

30. Woo HG, Wang XW, Budhu A, Kim YH, Kwon SM, Tang ZY, Sun Z, Harris CC and Thorgeirsson SS: Association of TP53 mutations with stem cell-like gene expression and survival of patients with hepatocellular carcinoma. Gastroenterology 140: 1063-1070, 2011.

31. Romei C and Elisei R: RET/PTC translocations and clinico-pathological features in human papillary thyroid carcinoma. Front Endocrinol 3: 54, 2012.

32. Kohno T, Ichikawa H, Totoki Y, Yasuda K, Hiramoto M, Nammo T, Sakamoto H, Tsuta K, Furuta K, Shimada Y, et al: $K I F 5 B-R E T$ fusions in lung adenocarcinoma. Nat Med 18: 375-377, 2012.

33. Zeng Q, Cheng Y, Zhu Q, Yu Z, Wu X, Huang K, Zhou M, Han S and Zhang Q: The relationship between overexpression of glial cell-derived neurotrophic factor and its RET receptor with progression and prognosis of human pancreatic cancer. J Int Med Res 36: 656-664, 2008.

34. Plaza-Menacho I, Morandi A, Robertson D, Pancholi S, Drury S, Dowsett M, Martin LA and Isacke CM: Targeting the receptor tyrosine kinase RET sensitizes breast cancer cells to tamoxifen treatment and reveals a role for RET in endocrine resistance. Oncogene 29: 4648-4657, 2010.

35. Phay JE and Shah MH: Targeting RET receptor tyrosine kinase activation in cancer. Clin Cancer Res 16: 5936-5941, 2010.

36. Fusco A, Grieco M, Santoro M, Berlingieri MT, Pilotti S, Pierotti MA, Della Porta G and Vecchio G: A new oncogene in human thyroid papillary carcinomas and their lymph-nodal metastases. Nature 328: 170-172, 1987.

37. Grieco M, Santoro M, Berlingieri MT, Melillo RM, Donghi R, Bongarzone I,Pierotti MA,Della Porta G, Fusco A and Vecchio G: PTC is a novel rearranged form of the ret proto-oncogene and is frequently detected in vivo in human thyroid papillary carcinomas. Cell 60: 557-563, 1990

38. Takeuchi K, Soda M, Togashi Y, Suzuki R, Sakata S, Hatano S, Asaka R, Hamanaka W, Ninomiya H, Uehara H, et al: RET, ROS1 and ALK fusions in lung cancer. Nat Med 18: 378-381, 2012. 